\title{
Prevalencia de anemia en la parroquia San Miguel
}

\author{
Jacinto Eugenio Pérez Ramírez \\ eugenioperezr@hotmail.com \\ Enma Jacqueline Zambrano Párraga \\ emmazam23@gmail.com \\ Catalina María Hurtado \\ bunayenterprises@gmail.com \\ Susan Karina Ortega Castillo \\ ortegasusan281@gmail.com \\ Jessica Ximena Humala Rojas \\ jessyhumala99@gmail.com \\ María Alejandra Mantilla Vicuña \\ alemantilla99@gmail.com \\ Pamela Lisset Pinagorte Santana \\ pamelapinargotes@gmail.com \\ Angie Patricia Mogrovejo Coronel \\ angiemogrovejo5658@gmail.com \\ Facultad de Medicina Universidad Católica de Cuenca Sede Azogues \\ Azogues - Ecuador
}

\section{RESUMEN}

La anemia a sido considerada como un problema que es parte de la salud pública afectando a los países en vías de desarrollo o tercermundistas, esta patología puede afectar en todas las edades, pero su mayor concentración se da en las edades tempranas. En Ecuador la prevalencia de la misma sigue siendo alta; la causa principal de anemia en a la niñez de debe al déficit de micronutrientes concretamente el hierro. El objetivo de este estudio fue determinar la prevalencia de anemia. Los resultados que se obtuvieron mostraron que de la población estudiada $52 \%$ fueron mujeres y el $48 \%$ hombres, además que la prevalencia de la anemia fue del $58 \%$, encontrándose una relación con la edad y el sexo de los niños y niñas. Se concluyó que la anemia se encuentra con mayor frecuencia en las mujeres y se presenta a menor edad.

Palabras clave: anemia; prevalencia; niñez, hierro; déficit. 


\title{
Prevalence of anemia in the San Miguel Parish
}

\begin{abstract}
Anemia has been considered as a problem that is part of public health affecting developing countries or third world countries, this pathology can affect all ages, but its highest concentration occurs in early ages. In Ecuador the prevalence of this disease is still high; the main cause of anemia in childhood is due to the déficit of micronutrients, especially iron. The objective of this study was to determine the prevalence of anemia. The results obtained showed that $52 \%$ of the population studied were female and $48 \%$ male, and that the prevalence of anemia was $58 \%$, with a relationship with the age and sex of the children. It was concluded that anemia is found more frequently in women and occurs at a younger age.
\end{abstract}

Keywords: anemia; prevalence; childhood; iron deficiency; iron deficiency.

Artículo recibido: 02 Setiembre. 2021 Aceptado para publicación: 30 Setiembre. 2021 Correspondencia: eugenioperezr@hotmail.com Conflictos de Interés: Ninguna que declarar 


\section{INTRODUCCIÓN}

La definición de anemia brindada por la Organización Mundial de la Salud (OMS) en niños en edad preescolar, hace referencia a una concentración de hemoglobina menor a $11 \mathrm{~g} / \mathrm{dl}$. Se estima que afecta a 253 millones correspondientes al 42,6\% de niños en esta edad.(AlKassab-córdova, Méndez-Guerra, \& Robles-Valcarcel, 2020)

La anemia se presenta como un problema de escala mundial golpeando a los países de bajos y medianos recursos; es así que en África y Asia se encuentran alrededor de un $45 \%$ de casos. (Chowdhury et al., 2020); mientras tanto, el $46 \%$ en el Mediterráneo Oriental y el $20 \%$ en el continente americano, europeo y la zona del Pacífico Occidental.(Moyan- Brito et al., 2020)

Las cifras brindadas por el Banco Mundial para América Latina y el Caribe, reflejan que cerca de 7,2 millones de niños menores de 5 años presentan una alteración o retraso del crecimiento, y 22,5 millones se reportan como anemia. La prevalencia de anemia y desnutrición crónica se encuentra entre 6 y 24 meses de edad.(Puma Chambi, Noemi; Azaña Laura, 2020)

En América latina la prevalencia de anemia esta entre el $5.1 \%$ en Chile en comparación con Haití donde sus cifras son del $45.5 \%$, es por ello que la misma se considera un problema dentro de la Salud Pública; pero que sucede en Bolivia encontramos hasta un $61 \%$ de niños y niñas con esta patología.(Garrido-Salazar, Garrido-Salazar, \& Vivas-Armas, 2019)

Pero que sucede en Ecuador, la encuesta nutricional ENSANUT-ECU realizada entre el 2012 reveló en un cuarto de siglo después la tasa de anemia era del $25.7 \%$, frente a la encuesta DANS realizada en 1986 que arrojo como resultados una prevalencia del $20.8 \%$ entre los 6 y 59 meses.(Ruiz \& Betancourt, 2020)

En Ecuador $70 \%$ de menores de 1 año sufren de anemia por déficit de hierro, dichas cifras que aumentan en la población rural. El hierro se considera un micronutriente esencial que ayuda a la producción de la hemoglobina, debido a que capta el oxígeno. Los niños tienen mayor predisponían de padecer anemia, debido <:a múltiples factores entre ellos el crecimiento y a la alta demanda del mismo.(Moyan- Brito et al., 2020)

Cerca del 34\% de la población mundial sufre de las consecuencias del déficit alimenticio, situación que dentro de la epidemiologia que ha sido bautizada como el "hambre oculta"; los micronutrientes reconocidos son el hierro, el iodo, y la vitamina A.(Ruiz \& Betancourt, 2020). 
Son múltiples los factores predisponentes para el desarrollo de anemia durante la niñez, las deficiencias esenciales de nutrientes, presencia de parásitos, nivel socioeconómico, déficit de lactancia materna, nivel de instrucción de la madre.(Al-Kassab-córdova et al., 2020) Durante la primera infancia, varios niños y niñas reciben alimentos pobres no solo en hierro sino también en calorías; de los cuales solo el $40 \%$ refieren el consumo de cárnicos.(Favero \& Rens, 2020)

El grupo etario más afectado lo constituyen los niños menores de 12 meses de edad; pero se aprecia que al aumentar la edad disminuye su prevalencia, es importante recalcar que cronicidad de la misma provoca: crecimiento retardado, alteraciones de tipo cognitivo que desencadenan disminución del aprendizaje.(Pediatría, 2018)

La anemia ferropénica corresponde al déficit de hierro que afecta a las mujeres en edad fértil, embarazadas, adolescencia, y los niños con edades entre menores 5 años; este déficit, es causal de retraso psicointelectual escolar, bajo peso al nacer y prematuridad.(Ruiz \& Betancourt, 2020)

No podemos olvidar las repercusiones de las enfermedades catalogadas como impacto en salud pública también económicas, en nuestro caso de estudio donde esta patología se asocia con una disminución del $0.5 \%$ del producto interno bruto de una nación.(Ruiz \& Betancourt, 2020)

Sin duda las complicaciones como el deterioro de la capacidad cognitiva, y el incremento de ciertas enfermedades además de la mencionada, podría representar el $4.1 \%$ del PIB del país.(Ruiz \& Betancourt, 2020)

Ante este hecho de impacto dentro de la salud pública es importante conocer la prevalencia de anemia en los niños y niñas en la parroquia rural de San Miguel del cantón Azogues, provincia del Cañar.

\section{ESTRATEGIAS METODOLÓGICAS}

Se ejecutó un estudio de tipo descriptivo transversal no experimental durante el año 2020 en la parroquia San Miguel del cantón Azogues, provincia del Cañar.

La población estudiada fue de 204 niños y niñas entre las edades de 6 y 59 meses, quienes acudieron a control del niño sano, donde se determinó el valor de hemoglobina.

Luego de la tabulación de datos se elaboraron tablas de frecuencias mediante la estadística descriptiva, finalmente se cruzaron las variables de estudio para determinar la relación mediante la prueba estadística chi cuadrado. 


\section{RESULTADOS Y DISCUSIÓN}

La base de datos estaba conformada por 204 niños y niñas entre los 6 y 59 meses, todos contaron con una cuantificación de hemoglobina.

La edad media fue 20.8 meses; de la población estudiada 52\% fueron mujeres y el $48 \%$ hombres, y el 100\% vivían en una zona rural.

La prevalencia de la anemia fue del $58 \%$ de la muestra mientras que el $42 \%$ no presentaron dicha patología; el $31 \%$ de los casos correspondieron a las mujeres y el $27 \%$ a los hombres. La edad donde existió mayor concentración fue entre los 6 y 15 meses de edad con el 28\%, y la de menor concentración se encontró desde los 46 a 59 meses con el 2.9\%.

En cuanto al análisis estadístico se obtuvo una relación entre la edad y el sexo con la anemia $(\mathrm{p}<0,01)$.

En un estudio realizado por Al-Kassab-córdova et al (2020) en Perú encontró una prevalencia de anemia del $38.5 \%$ entre las edades similares al estudio, además indica la relación entre la edad del niño y la presencia de anemia; como contraste versus nuestro estudio la población estudiada correspondía al $60.8 \%$ del casco urbano.

De igual forma un estudio realizado en Nepal Chowdhury et al (2020) encontró relación estadística $(\mathrm{p}<0.01)$ entre la edad del niño y anemia, es decir menor edad mayor probabilidad de desarrollar esta patología, con la diferencia que la población correspondió a un $53.5 \%$ de hombres donde se concentró la prevalencia además de que la población correspondía al $56.2 \%$ urbana.

Favero y Rens (2020) encontraron una prevalencia de anemia en un 50.6\% con mayor concentración entre los 6 y 12 meses de edad, además de que a menor edad aumenta riesgo de anemia por déficit de hierro, sin embargo, las características de población mostro escasa asociación.

Según lo detallado en el estudio realizado por Garrido-Salazar et al (2019) recalca que en la provincia del Cañar se encontró una prevalencia del 51. 8\%, además de que consideran a esta entidad como un problema de salud pública.

Finalmente, Solano Barquero et al (2018), encontraron una prevalencia alta en menores de 5 años, la misma que se concentró el sexo femenino, recalcan que este estadio se debe al déficit nutricional, y a características socioeconómicas, además del cuidado de menores.

\section{CONCLUSIÓN O CONSIDERACIONES FINALES}


La prevalencia de anemia durante el año 2020 fue del 58\% y correspondió en su gran mayoría a las mujeres, además se asociaron las variables como la edad y sexo con la presencia de esta patología.

La población rural se ve en desigualdad sin un abordaje oportuno sobre las diferentes determinantes de la salud.

Se deben plantear estrategias que garanticen el acceso y la reducción de esta patología en la niñez.

Ampliar las variables de estudio como: factores socioeconómicos, tipo de alimentación del niño o niña, estado nutricional mediante antropometría, ente otras.

Fortalecer actividades encaminadas a la promoción de la salud mediante talleres sobre prevención de la anemia en la niñez.

\section{LISTA DE REFERENCIAS}

Al-Kassab-córdova, A., Méndez-Guerra, C. I., \& Robles-Valcarcel, P. (2020). Sociodemographic and nutritional factors associated with anemia in children aged 1 to 5 years old in Peru. Revista Chilena de Nutricion, 47(6), 925-932. https://doi.org/10.4067/S0717-75182020000600925

Amano, I., \& Murakami, A. (2019). Prevalence of infant and maternal anemia during the lactation period in Japan. Pediatrics International, 61(5), 495-503. https://doi.org/10.1111/ped.13833

Chowdhury, M. R. K., Khan, M. M. H., Khan, H. T. A., Rahman, M. S., Islam, M. R., Islam, M. M., \& Billah, B. (2020). Prevalence and risk factors of childhood anemia in Nepal: A multilevel analysis. PLoS ONE, 15(10 October). https://doi.org/10.1371/journal.pone.0239409

De Paz, R., Canales, M., \& Hernández, F. (2006). Anemia ferropénica. Medicina Clinica, 127(3), 100-103. https://doi.org/10.1157/13090266

Durá Travé, T., \& Díaz Vélaz, L. (2002). Prevalencia de la deficiencia de hierro en lactantes sanos de 12 meses de edad. Anales Espanoles de Pediatria, 57(3), 209-214. https://doi.org/10.1016/s1695-4033(02)77907-1

Favero, M., \& Rens, V. (2020). Anemia y déficit de hierro en lactantes de 6 a 12 meses de la ciudad de Necochea: prevalencia y determinantes. Archivos Argentinos de Pediatria, 118(3), 187-192. https://doi.org/10.5546/aap.2020.187

Fernández, A. S., Díaz, R. D. S., \& Verdecia, O. A. (2017). Caracterización de lactantes 
menores de 6 meses con anemia ferropénica. Revista Cubana de Pediatria, 89(1), 12 19.

Garrido-Salazar, D. I., Garrido-Salazar, S. M., \& Vivas-Armas, G. (2019). Anemia frequency in children living at Andean high altitude in Ecuador, Peru, and Bolivia. Acta Pediatrica de Mexico, 40(6), 305-317. https://doi.org/10.18233/apm40no6pp305-3171929

Hernández, A. (2012). Anemias en la infancia y adolescencia. Clasificación y diagnóstico. Pediatria Integral, XVI(5), 357-365.

Hospital, I., Integrada, S., Hospital, I., \& Hospital, I. (2016). Artículo aceptado Artículo aceptado.

López-Huamanrayme, E., Atamari-Anahui, N., Rodriguez-Camino, M. C., Mirano-Ortizde-Orue, M. G., Quispe-Cutipa, A. B., Rondón-Abuhadba, E. A., \& Pereira-Victorio, C. J. (2019). Prácticas de alimentación complementaria, características sociodemográficas y su asociación con anemia en niños peruanos de 6-12 meses. Rev. Habanera Cienc. Méd, 18(5), 6-12.

Machado, K., Alcarraz, G., Morinico, E., Briozzo, T., Gutiérrez, S., Machado, K., ... Gutiérrez, S. (2017). Archivos de pediatría del Uruguay órgano oficial de la Sociedad Uruguaya de Pediatría. Archivos de Pediatría Del Uruguay, 88(5), 254-260. Retrieved from http://www.scielo.edu.uy/scielo.php?script=sci_arttext\&pid=S168812492017000500254

Mahoney, D. (2016). La deficiencia de hierro en lactantes y niños pequeños: detección, prevención, manifestaciones clínicas y diagnóstico. UpToDate, 1-21. Retrieved from https://www.uptodate.com/contents/iron-deficiency-in-infants-and-young-childrenscreening-prevention-clinical-manifestations-and-

diagnosis?source=see_link\&sectionName=Presumptive diagnosis and empiric trial\&anchor=H19509670\#H19509670

Martínez, O., \& Baptista, H. (2019). Anemia por deficiencia de hierro en niños: un problema de salud nacional. Rev Hematol Mex. 2019 Abril-Junio, 20(2), 96-105. Retrieved from https://doi.org/10.24245/rhematol.

Moyan- Brito, E., Vintimilla-Molina, J., Calderón-Guaraca, P., Parra-Pérez, C., AyoraCambisaca, E., \& Angamarca-Orellana, M. (2020). Factores asociados a la anemia en niños ecuatorianos de 1 a 4 años. AVFT-Archivos Venezolanos de Farmacología y 
Terapéutica, 38(6), 695-699.

Muñoz Del Carpio-Toia, Á., Cornejo-Roselló, I., Rojas-Pauca, S., Alvarez-Cervantes, G., Bernabé-Ortiz, J. C., Gallegos, A., ... Toia-Larsen, M. (2020). Anemia infantil en poblaciones que residen a diferentes altitudes geográficas de Arequipa, Perú: estudio $\begin{array}{llll}\text { descriptivo } & \text { y } & \text { Metrospectivo. } & \text { 20(7), }\end{array}$ https://doi.org/10.5867/medwave.2020.07.8004

Pediatría, R. C. De. (2018). Revista Cubana de Pediatría, Vol. 90, No. 4 (2018). 90(4).

Picos Nordet, S., Santiesteban González, B. de la C., Cortés Santos, M. del C., Morales Gómez, A. C., \& Acosta Alegría, M. (2015). Risk factors in the onset of anemia in 6 months-old infants. Rev. Cuba. Pediatr, 87(4), 404-412.

Pittman, Q. J. (2020). A gut feeling about the ketogenic diet in epilepsy. Epilepsy Research, 166. https://doi.org/10.1016/j.eplepsyres.2020.106409

Puma Chambi, Noemi; Azaña Laura, V. (2020). Universidad peruana union. Dirección General de Investigación, 1-93. Retrieved from https://drive.google.com/file/d/1_1R8G2xOKhcG-2YVMyc1XXEeJ99GQQ2s/view

Quizhpe, E., San Sebastián, M., Hurtig, A. K., \& Llamas, A. (2003). Prevalencia de anemia en escolares de la zona Amazónica de Ecuador. Revista Panamericana de Salud Publica/Pan American Journal of Public Health, 13(6), 355-361. https://doi.org/10.1590/s1020-49892003000500003

Ruiz, P., \& Betancourt, S. (2020). Sobre la anemia en las edades infantiles en el Ecuador causas e intervenciones correctivas y preventivas. Revista Cubana de Alimentación y Nutricion 30(1), 218-235. Retrieved from https://www.medigraphic.com/pdfs/revcubalnut/can-2020/can201o.pdf

Sánchez Ruiz-Cabello, F. J. (2012). Prevención y cribado de la ferropenia en lactantes.

Pediatría Atención Primaria, 14(53), 75-82. https://doi.org/10.4321/s113976322012000100013

STEGEN, G., JONES, K., BARROS, M., FADDA, F., FREISTADT, K., CAMPODOnico, M., \& PASTENE, M. (1963). Ferropenia. Revista Chilena de Pediatría, 34, 426-428. 